\title{
Genome informatics and vaccine targets in Corynebacterium urealyticum using two whole genomes, comparative genomics, and reverse vaccinology
}

Luis Carlos Guimarães', Siomar de Castro Soares², Eva Trost ${ }^{3}$, Jochen Blom ${ }^{4}$, Rommel Thiago Jucá Ramos ${ }^{5}$, Artur Silva ${ }^{5}$, Debmalya Barh', Vasco Azevedo ${ }^{1 *}$

From X-meeting 2014 - International Conference on the Brazilian Association for Bioinformatics and Computational Biology

Belo Horizonte, Brazil. 28-30 October 2014

\begin{abstract}
Background: Corynebacterium urealyticum is an opportunistic pathogen that normally lives on skin and mucous membranes in humans. This high Gram-positive bacteria can cause acute or encrusted cystitis, encrusted pyelitis, and pyelonephritis in immunocompromised patients. The bacteria is multi-drug resistant, and knowledge about the genes that contribute to its virulence is very limited. Two complete genome sequences were used in this comparative genomic study: C. urealyticum DSM 7109 and C. urealyticum DSM 7111.

Results: We used comparative genomics strategies to compare the two strains, DSM 7109 and DSM 7111, and to analyze their metabolic pathways, genome plasticity, and to predict putative antigenic targets. The genomes of these two strains together encode 2,115 non-redundant coding sequences, 1,823 of which are common to both genomes. We identified 188 strain-specific genes in DSM 7109 and 104 strain-specific genes in DSM 7111. The high number of strain-specific genes may be a result of horizontal gene transfer triggered by the large number of transposons in the genomes of these two strains. Screening for virulence factors revealed the presence of the spaDEF operon that encodes pili forming proteins. Therefore, spaDEF may play a pivotal role in facilitating the adhesion of the pathogen to the host tissue. Application of the reverse vaccinology method revealed 19 putative antigenic proteins that may be used in future studies as candidate drug or vaccine targets.

Conclusions: The genome features and the presence of virulence factors in genomic islands in the two strains of C. urealyticum provide insights in the lifestyle of this opportunistic pathogen and may be useful in developing future therapeutic strategies.
\end{abstract}

\section{Background}

The species Corynebacterium urealyticum was proposed in 1986, but this bacteria was first isolated between June 1983 and March 1984, when four patients were diagnosed with alkaline-encrusted cystitis. The published

\footnotetext{
* Correspondence: vasco@icb.ufmg.br

'Department of General Biology, Institute of Biological Sciences, Federal University of Minas Gerais, Avenue Antônio Carlos, 6627, Belo Horizonte, Minas Gerais, Brazil

Full list of author information is available at the end of the article
}

case report describes the isolates as belonging to the Corynebacterium group D2 [1,2]. Chemotaxonomic studies and $16 \mathrm{~S}$ rRNA sequence comparisons showed that $C$. urealyticum was more closely related to Corynebacterium lipophilic species such as Corynebacterium jeikeium, but could be differentiated from C. jeikeium based on its ability to hydrolyze urea [3]. C. urealyticum is a Gram-positive, non-spore-forming, aerobic, and slow-growing bacteria. Its cell wall is composed of peptidoglycan, menaquinone, mycolic acids, and cellular fatty 
acids, which is the common composition of the cell walls of Corynebacterium species [4].

C. urealyticum is an opportunistic pathogen commonly isolated from the skin and mucous membranes of hospitalized patients. The pathogen mainly causes acute or encrusted cystitis, encrusted pyelitis, and pyelonephritis [4]. Its urease activity is the main factor that contributes to the ability of $C$. urealyticum to colonize the urinary tract where its presence is associated with alkaline $\mathrm{pH}$ and the formation of ammonium magnesium phosphate stones $[1,2]$. C. urealyticum is a multi-drug resistant bacterium and its treatment requires the administration of multiple drugs and additional invasive interventions $[4,5]$. Currently, the complete genome sequences of only two C. urealyticum strains, DSM 7109 and DSM 7111, are publicly available. In these two genomes, the antibiotic resistance genes were located in mobile DNA, suggesting that the multidrug resistance was acquired through horizontal gene transfer [6,7].

In this work, we compared the genome sequences of the two $C$. urealyticum multidrug resistance strains DSM 7109 and DSM 7111, focusing on differences in the gene content and metabolic pathways between the two strains. We also attempted to identify new candidate targets that can be used in the development of drugs or vaccines against this pathogen.

\section{Methods}

\section{Genome sequences of $C$. urealyticum strains DSM 7109 and DSM 7111}

C. urealyticum DSM 7109 was isolated from a patient with alkaline-encrusted cystitis and C. urealyticum DSM 7111 was isolated from the urine samples of a 9-year-old patient with an ectopic kidney. The genome sequences of both these strains were retrieved from the NCBI GenBank database (http://www.ncbi.nlm.nih.gov/genbank/) [8] [GenBank:NC_010545.1, GenBank: NC_020230.1].

\section{Bioinformatics analysis}

The origin of chromosomal DNA replication (oriC) gene was predicted using the Ori-Finder web program [9]. The Ori-Finder prediction was based on a combined process: (i) gene identification involving analysis of base composition through Z-curve method; and, (ii) occurrence of genes frequently close to oriCs (distribution of $d n a A$ boxes along the genome) [9]. The CRISPRs were predicted using the CRISPRfinder web program [10]. These regions are important because they confer protection against bacteriophages. The comparative analysis was done using the EDGAR web-program that compares genome content based on the calculation of the BLAST score ratio by automatically adjusted cutoff for each selected dataset [11].

\section{In silico identification metabolic pathway construction}

The metabolic pathways reconstruction of $C$. urealyticum was performed using the genome sequence file in FASTA format and the genome annotation file in GBK format. Metabolic pathways databases for strains DSM 7109 and DSM 7111 were created using the Pathway Tools 13 software (available at http://bioinformatics.ai. sri.com/ptools/), developed by SRI International [12]. The Pathway Tools software contains algorithms that can predict the metabolic pathways of an organism from its genome by comparing it to a reference pathways database known as the MetaCyc Database [13]. Construction of a metabolic pathways database was done using the BioCyc collection [14].

\section{Prediction of genome plasticity of C. urealyticum DSM 7109 and DSM 7111 strains}

Prediction of genomic islands in C. urealyticum genomes was done using PIPS software [15]. PIPS detects genome signatures like $C+G$ content, codon usage deviation, high concentrations of virulence factors, hypothetical proteins, the presence of transposases and tRNA flanking sequences, and absence of query regions in non-pathogenic organisms. C. glutamicum ATCC 13032 was used as the closely related non-pathogenic species to C. urealyticum in PIPS. The BRIG software [16] was used for plasticity comparisons among C. urealyticum (DSM 7109 and DSM 7111 strains), C. pseudotuberculosis 1002, C. diphtheriae NCTC 13129, C. ulcerans 809, and C. glutamicum ATCC 13032.

\section{Prediction of putative antigenic targets of $C$. urealyticum}

To identify antigenic targets, we used the strategy described by Barh et al. [17] with modifications. We also adopted the four rules as per the reverse vaccinology strategy of Rappuoli et al. [18] for final selection of the putative vaccine targets. Rule I: consider the antigenic proteins that are either secreted proteins, surfaceexposed proteins, or membrane proteins so that they can be exposed to the host, and therefore can be promptly recognized by the host immune system [18]; Rule II: major histocompatibility complex (MHC) I and II binding properties with adhesion probability greater than 0.51 and absence of similarity to host proteins [19]; Rule III: protein conservation among different genomes [19]; and Rule IV: virulence factors are normally encoded within genomic islands [18]. Rule IV does not exclude the targets from Rule III.

SurfG + software [20] was used to predict targets according to the Rule I. This software classifies proteins according to their subcellular location using the presence or absence of signal peptides, retention signals, and transmembrane helices. To apply Rule II, the 
proteins predicted by surfG + were analyzed using the Vaxign software [19]. Because the aim of this work was to identify vaccine candidates, the predicted proteomes were screened for proteins that were potentially antigenic in both strains (Rule III). To achieve this, we used the Artemis Comparison Tool [21] with BLAST alignment comparison files and searched for antigenic proteins that show more than $70 \%$ similarity in $70 \%$ of their extensions in both strains. Base on Rule IV, we screened the detected antigenic proteins for antigenic targets harbored by shared genomic islands in the two strains.

\section{Results and discussion}

\section{Genomic architecture and features of $C$. urealyticum} strains DSM 7109 and DSM7111

Strains DSM 7109 and DSM 7111 were isolated from patients with alkaline-encrusted cystitis [6,7]. The genomic composition of these two strains is very similar; i.e., both sequences have the same $\mathrm{G}+\mathrm{C}$ content, coding density, ribosomal RNAs clusters, and clustered regularly interspaced short palindromic repeats (CRISPRs). However, the sizes of the two genomes are different: the DSM 7111 genome contains 2,316,065 bp and is $50 \mathrm{~Kb}$ smaller than the DSM 7109 genome with 2,369,219 bp. As expected, the number of coding sequences in the DSM 7111 genome (1,927 protein coding regions) is lower than in the DSM 7109 genome (2,011 protein coding regions) because both genomes have the same coding density. These data indicate a strain-specific difference in the gene repertories in both these isolates. Relevant data and general features from both genome sequences are summarized in Table 1.

Our analysis of the GC skew $[(\mathrm{G}-\mathrm{C}) /(\mathrm{G}+\mathrm{C})]$ revealed that both genome sequences contained a bi-directional replication mechanism (Figure 1). The origin of the chromosomal replication (oriC) gene is located between the replication initiator genes $d n a A$ (downstream) and dnaN (upstream) and has a size of $843 \mathrm{bp}$. However, the G/C skew analysis did not confirm that the replication termination site, dif, was located at the $180^{\circ}$ position from oriC $[9,22]$.

CRISPRs are often associated with cas genes that normally provide resistance against bacteriophages [23].

Table 1. General features of the genomes of C. urealyticum strains DSM 7109 and DSM 7111

\begin{tabular}{lcc}
\hline Feature & DSM 7109 & DSM 7111 \\
\hline Genome size (bp) & $2,369,219$ & $2,316,065$ \\
C+G content (\%) & 64.2 & 64.2 \\
Coding sequences & 2,084 & 2,007 \\
Coding density (\%) & 90.16 & 89.53 \\
ribosomal RNAs & $3 \times(16 S-23 S-5 S)$ & $3 \times(16 S-23 S-5 S)$ \\
transfer RNAs & 51 & 54 \\
\hline
\end{tabular}

One CRISPR region was predicted in each genome (strains DSM 7109 and DSM 7111) using the CRISPRFinder software [10]. Both these regions were flanked by seven cas genes. The size of one CRISPR was $28 \mathrm{bp}$ and the consensus sequence was the same for the CRISPRs in both genomes. Each CRISPR was separated by 69 bp (Table 2).

\section{Gene sharing among the two $C$. urealyticum strains}

Orthologous genes were detected using the EDGAR software, which defines subsets of genes using the SRV method to predict orthologous genes in prokaryotic genomes [11]. We found that the DSM 7109 and DSM 7111 genomes together encode 2,115 no-redundant coding gene sequences; $1,823(86.2 \%)$ of these coding gene sequences were common to both strains, and 188 and 104 were specific to DSM 7109 and DSM 7111, respectively. Species-specific genes have been linked to niche adaptation of microorganisms. A previous study of 17 Escherichia coli strains found that less than $50 \%$ of the genes (2,200 genes in a total of 5,000 genes) were shared among these strains [24]. Therefore, we can infer that the DSM 7109 and DSM 7111 genomes are very similar because they share a high proportion of their genes. A previous study of four Corynebacterium pseudotuberculosis strains [25] also reported a large number in shared genes; 1,851 (77.9\%) genes in a total of 2,377 genes. Clearly, the numbers of core genes are likely to reduce when more strains of $C$. urealyticum are added, as was shown previously by Soares and colleagues in a study of 15 C. pseudotuberculosis strains were 1,504 $(54.5 \%)$ genes in a total of 2,782 genes were shared [26].

\section{Plasticity of the C. urealyticum DSM 7109 and DSM 7111 genomes}

Genome plasticity has been used to provide insights into genome evolution through the study of horizontally acquired genomic regions. The transfer of blocks of genes (genomic islands) normally correlates with the acquirement of a given function, like virulence (pathogenicity islands), degradation of secondary compounds (metabolic islands), antibiotic resistance (resistance islands) and symbiotic relationships with Leguminosae (symbiotic islands)[15]. Furthermore, because genomic islands are acquired from a different organism, they are responsible for deviations in genomic signatures such as codon usage and $\mathrm{G}+\mathrm{C}$ content once they reflect the genomic signature of the donor organism [27]. We used the PIPS software [15] to predict genomic islands in both $C$. urealyticum strains. Twenty-six genomic islands were predicted in each genome (Figure 1). DSM 7109 had 556 genes present in the genomic islands and DSM 7111 had 496 genes. We identified 403 genes in the genomic islands that belonged to the shared genes 


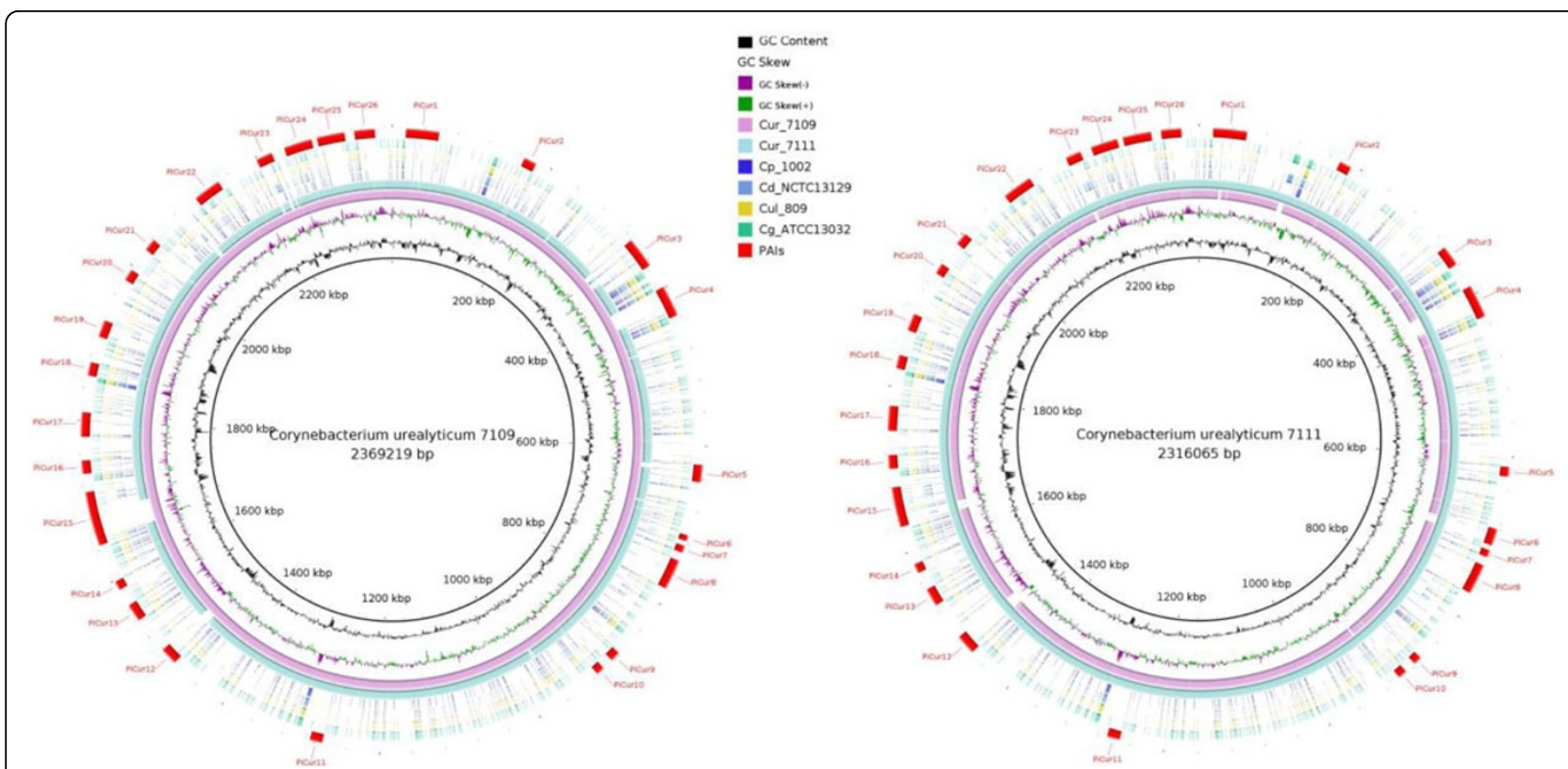

Figure 1 Comparative genomic maps of C. urealyticum strains DSM 7109 and DSM 7111. The C. urealyticum DSM 7109 map (left) used as the reference. The $C$. urealyticum DSM 7111 map (right) used as the reference. From the inner to outer circles on the genome maps: $C$. urealyticum DSM 7109 (Cur_7109) then C. urealyticum DSM 7111 (Cur_7111) for the map on the left; C. urealyticum DSM 7111 (Cur_7111) then C. urealyticum DSM 7109 (Cur_7109) for the map on the right; followed by C. pseudotuberculosis 1002 (Cp_1002), C. diphtheriae NCTC 13129 (Cd_NCTC13129), C. ulcerans 809 (Cul_809), C. glutamicum ATCC 13032 (Cg_ATCC13032). The outermost circles in both maps indicate the 26 genomic islands (PiCur 1-26).

dataset, meaning that the majority of genes that were acquired by horizontal gene transfer were commonly shared genes.

Previous studies on genomic islands in C. pseudotuberculosis and $C$. diphtheriae identified 16 and 52 genomic islands, respectively $[26,28]$. Only nine of the genes in the genomic islands were shared among $C$. urealyticum strains DSM 7109 and DSM 7111, C. pseudotuberculosis 1002, and C. diphtheriae NCTC 13129 (Table 3). The low number of shared genes among different species in the same genus is expected because their habitats are different and genomic islands are normally acquired through horizontal gene transfer as was shown previously by Perrin and colleagues [29]. Pathogenicity islands contain genes correlated with virulence [27]. Therefore, these genes may be good candidates for the development of vaccines or drugs [15]. When we compared the orthologs of the candidate virulence factors reported in C. ulcerans [30] with the genes in the pathogenicity islands in C. urealyticum we found only one common gene and this gene was annotated with unknown function (ID: CU7111_1212 for DSM 7111; ID: cur_1230 for DSM 7109).

Table 2. Structural features of CRISPR loci predicted in C. urealyticum strains DSM 7109 and DSM 7111

\begin{tabular}{|c|c|c|c|c|c|c|}
\hline Strain & $\begin{array}{l}\text { No. of CRISPR } \\
\text { loci }\end{array}$ & $\begin{array}{l}\text { No. of cas } \\
\text { genes }\end{array}$ & $\begin{array}{l}\text { locus_tag cas } \\
\text { genes }\end{array}$ & $\begin{array}{c}\text { No. of } \\
\text { spacers }\end{array}$ & $\begin{array}{l}\text { CRISPR } \\
\text { size }\end{array}$ & ${ }^{*}$ CRISPR consensus sequence \\
\hline $\begin{array}{c}\text { Corynebacterium urealyticum } \\
\text { DSM } 7109\end{array}$ & 1 & 7 & $\begin{array}{l}\text { cur_1967 } \\
\text { cur_1968 } \\
\text { cur_1969 } \\
\text { cur_1970 } \\
\text { cur_1971 } \\
\text { cur_1972 } \\
\text { cur_1973 }\end{array}$ & 69 & $28 \mathrm{bp}$ & GGCTCATCCCCGCTGGCGCGGGGAGCAC \\
\hline $\begin{array}{c}\text { Corynebacterium urealyticum } \\
\text { DSM } 7111\end{array}$ & 1 & 7 & $\begin{array}{l}\text { CU7111_1887 } \\
\text { CU7111_1888 } \\
\text { CU7111_1889 } \\
\text { CU7111_1890 } \\
\text { CU7111_1891 } \\
\text { CU7111_1892 } \\
\text { CU7111_1893 }\end{array}$ & 69 & $28 \mathrm{bp}$ & GGCTCATCCCCGCTGGCGCGGGGAGCAC \\
\hline
\end{tabular}


Table 3. Genes present in genomic islands shared by related Corynebacterium species

\begin{tabular}{|c|c|c|c|c|c|}
\hline $\begin{array}{c}\text { C. urealyticum DSM } \\
7109\end{array}$ & $\begin{array}{c}\text { C. urealyticum DSM } \\
7111\end{array}$ & $\begin{array}{c}C . \\
\text { pseudotuberculosis } \\
1002\end{array}$ & $\begin{array}{c}\text { C. diphtheria NCTC } \\
13129\end{array}$ & $\begin{array}{l}\text { Gene } \\
\text { name }\end{array}$ & Product \\
\hline cur_1756 & CU7111_1693 & Cp1002_1932 & DIP2331 & - & Putative aldehyde dehydrogenase \\
\hline cur_1817 & CU7111_1751 & Cp1002_1909 & DIP2133 & - & Fe-S oxidoreductase \\
\hline cur_1897 & CU7111_1828 & Cp1002_1870 & DIP0236 & $s r t B$ & $\begin{array}{l}\text { Fimbrial associated sortase (Surface protein } \\
\text { transpeptidase) }\end{array}$ \\
\hline cur_1898 & CU7111__1829 & Cp1002_1872 & DIP0235 & spaD & $\begin{array}{l}\text { Putative surface-anchored protein (Fimbrial } \\
\text { subunit) }\end{array}$ \\
\hline cur_1899 & CU7111_1830 & Cp1002_1874 & DIP0233 & srtc & $\begin{array}{l}\text { Fimbrial associated sortase (Surface protein } \\
\text { transpeptidase) }\end{array}$ \\
\hline cur_1933 & CU7111_1856 & Cp1002_0132 & DIP0247 & $\operatorname{tad} A$ & tRNA-specific adenosine deaminase \\
\hline cur_1934 & CU7111_1857 & Cp1002_0131 & DIP0246 & - & Hypothtical protein \\
\hline cur_1935 & CU7111_1858 & Cp1002_0130 & DIP0245 & tyrA & Prephenate dehydrogenase \\
\hline cur_1939 & CU7111_1861 & Cp1002_0120 & DIP0179 & - & Putative dicarboxylate uptake system \\
\hline
\end{tabular}

The genomes of C. urealyticum strains DSM 7109 and DSM 7111, C. pseudotuberculosis 1002, and C. diphtheriae NCTC 13129 were compared.

Prediction of candidate vaccine targets for $C$. urealyticum The sub-cellular location of proteins in DSM 7109 and DSM 7111 was predicted using the SurfG + software [31], which classifies genes into four categories: cytoplasmic, membrane, PSE (putative surface-exposed), and secreted (Table 4). We used the four rules described in the reverse vaccinology strategy (see the Methods section for details) for final selection of putative vaccine targets. According to Rule I, proteins exposed to the host are better candidates because they can be promptly recognized by the immune system; for example, secreted proteins, surface-exposed proteins, and membrane proteins. We predicted 590 and 579 putative candidates for DSM 7109 and DSM 7111, respectively, using Rule I. The encoded proteins were submitted to the Vaxign software [19], which detected 54 and 57 proteins with antigenic properties in DSM 7109 and DSM 7111, respectively. Using Rule III, we considered only proteins that were shared by both strains, which resulted in 46 candidates for both strains. Finally, using Rule IV, we identified 19 proteins that were shared by both strains and that were encoded within genomic islands as vaccine candidates (Table 5). Among these 19 vaccine candidates, six were annotated with a function and a gene name. These proteins have been identified as potential vaccine targets in previous studies [30,32-36], but as yet no tests have been carried out to confirm this.

Table 4. Subcellular location of proteins from C. urealyticum strains DSM 7109 and DSM 7111

\begin{tabular}{lcc}
\hline Feature & DSM 7109 & DSM 7111 \\
\hline Cytoplasmic proteins & 1431 & 1356 \\
Membrane proteins & 311 & 302 \\
PSE $^{a}$ proteins & 200 & 198 \\
Secreted proteins & 79 & 79 \\
\hline
\end{tabular}

${ }^{\text {a }}$ Putative surface-exposed
The $r p f C$ gene (resuscitation-promoting factor) is a member of a protein family ( $r p f A, r p f B, r p f D$, and $r p f E)$ found in Actinobacteria. The protein encoded by $r p f C$ plays a role in stimulating resuscitation of dormant cells and in the multiplication of normal viable bacteria. Studies in Mycobacterium luteus with a disrupted rpf gene were not possible because of the absence of a second functional copy of the gene, suggesting that this protein is essential for normal growth and reduces the lag phase of diluted fast-growers [32,37].

The mepA gene (penicillin-insensitive murein endopeptidase) in $E$. coli encodes a protein the cleaves the D-alanyl-meso-2,6-diamino-pimelyl amide bond of peptidoglycans [38]; however, this protein is sensitive to metal-chelating agents such as lipoteichoic acid and deoxyribonucleic acid [39]. Previous studies with metallopeptidases showed that animals infected with C. pseudotuberculosis reacted to the mepA protein, while non-infected animals did not. This protein has transmembrane domains, another strong indication that it may make a good molecular vaccine target $[33,40]$.

The lpps (lipoprotein) gene encodes a protein that is associated with cell envelopes and has four known lipoprotein functions: (i) structural function (murein lipoproteins); (ii) transport function (substrate-binding proteins of $\mathrm{ABC}$ transporters in Gram-positive bacteria); (iii) adhesion function; and (iv) enzymatic function. The lipoprotein present in C. urealyticum has an L,D-transpeptidase catalytic domain, which gives the bacteria the ability to resist beta-lactam antibiotics by inhibiting PBPs (penicillin-binding protein) $[34,41]$.

The $c m t A$ (trehalose corynomycol transferase) gene encodes a protein that has catalytic function. It plays a role in the transfer of mycolic acids through trehalose monocorynomycolate on the cell wall arabinogalactan to 
Table 5. Putative antigenic proteins identified using Vaxign and shared by two $C$. urealyticum strains

\begin{tabular}{ccccl}
\hline DSM 7109 - Locus_Tag & DSM 7111 - Locus_Tag & Gene name & Subcellular location & Gene product \\
\hline cur_0025 & CU7111_0027 & rpfC & secreted & Resuscitation-promoting factor RpfC \\
cur_0151 & CU7111_0157 & - & secreted & Putative secreted protein \\
cur_0291 & CU7111_0284 & - & PSE & Putative surface-anchored protein \\
cur_0295 & CU7111_0288 & - & PSE & Hypothetical protein \\
cur_0527 & CU7111_0510 & - & PSE & Putative secreted protein \\
cur_0530 & CU7111_0513 & mepA & secreted & Putative secreted metallopeptidase \\
cur_1309 & CU7111_1290 & - & PSE & Hypothetical protein \\
cur_1319 & CU7111_1300 & - & PSE & Putative ribonuclease \\
cur_1350 & CU7111_1330 & - & secreted & Putative secreted protein \\
cur_1399 & CU7111_1390 & IppS & PSE & Putative lipoprotein \\
cur_1604 & CU7111_1545 & - & secreted & Hypothetical protein \\
cur_1636 & CU7111_1577 & - & secreted & Putative secreted protein \\
cur_1834 & CU7111_1766 & - & PSE & Hypothetical protein \\
cur_1842 & CU7111_1775 & cmtA & secreted & Trehalose corynomycolyl transferase A \\
cur_1896 & CU7111_1827 & spaE & PSE & Putative surface-anchored protein (fimbrial subunit) \\
cur_1898 & CU7111_1829 & spaD & PSE & Putative surface-anchored protein (fimbrial subunit) \\
cur_1958 & CU7111_1880 & - & secreted & Hypothetical protein \\
cur_1959 & CU7111_1881 & - & secreted & Hypothetical protein \\
cur_1980 & CU7111_1900 & crCB & membrane & Putative fluoride ion transporter CrcB \\
\hline
\end{tabular}

another trehalose monocorynomycolate to produce trehalose dicorynomycolate [42].

The spaD and spaE genes are part of the spaDEF cluster that encodes adhesive pilus structures that are surfaceanchored to the cell walls of Corynebacterium where they probably facilitate the adhesion of the pathogen to the host tissue. We detected the spaF gene in the DSM 7109 and DSM 7111 genomes, as well as the sortase encoding genes $\operatorname{srt} B$ and $\operatorname{srt} C$. The genome organization of the spaDEF cluster in the two C. urealyticum strains is similar to cluster organization in C. diphitheriae NCTC 13129 and C. ulcerans 809 and BR-AD22 strains [30,43]. However, the spaABC cluster of genes proposed as an essential virulence factor in $C$. diphtheria [35] was absent in both strains of $C$. urealyticum analyzed.

The $\operatorname{crcB}$ gene encodes a putative membrane protein, important for the reducing the fluoride concentration in cells, thus reducing its toxicity. Fluoride ions reduce cell growth, even when present in millimolar concentrations. Thus, we can infer that $\operatorname{crc} B$ gene is an efficient resistance mechanism [36].

\section{Differences in metabolic pathways in the $C$. urealyticum genomes}

To predict the metabolic pathways encoded in the DSM 7109 and DSM 7111 genomes, we used the Pathway Tools software (version 13.0) [44], and detected 226 and 271 pathways in DSM 7109 and DSM 7111, respectively. We also identified 942 and 1,116 metabolic reactions for these strains (Table 6).
Comparative analysis of two pathway classes (Biosynthesis and Degradation/Utilization/Assimilation) showed that the DSM 7109 and DSM 7111 genomes had 139 and 174 Biosynthesis pathways, respectively (Table 7), which is quite different from the number of pathways we found previously in other species in the same genus; for example, C. pseudotuberculosis strains 1002 and C231 in which 105 and 104 Biosynthesis pathways were predicted, respectively [25]. The number of Degradation/Utilization/Assimilation pathways predicted in the DSM 7109 and DSM 7111 genomes where similar, 70 pathways in DSM 7109 and 66 pathways in DSM 7111 (Table 7).

On further analysis, we found that the DSM 7109 and DSM 7111 genomes had 25 and 57 unique metabolic pathways (Table S1, additional file 1), respectively, even though both the strains were isolated from humans and caused the same symptoms [6,7].

Table 6. Numbers of gene data types in C. urealyticum strains DSM 7109 and DSM 7111

\begin{tabular}{lcc}
\hline Data type & DSM 7109 & DSM 7111 \\
\hline Genes & 2082 & 1998 \\
Pathways & 226 & 271 \\
Metabolic reactions & 942 & 1116 \\
Transport reactions & 34 & 36 \\
Polypeptides & 2022 & 1935 \\
Enzymes & 536 & 543 \\
\hline
\end{tabular}


Table 7. Numbers of pathways in C. urealyticum strains DSM 7109 and DSM 7111

\begin{tabular}{|c|c|c|}
\hline Pathway class & DSM 7109 & DSM 7111 \\
\hline Biosynthesis & 139 & 174 \\
\hline -Amines and Polyamines Biosynthesis & 4 & 5 \\
\hline -Amino Acids Biosynthesis & 27 & 32 \\
\hline -Aminoacyl-tRNA Charging & 3 & 3 \\
\hline -Aromatic Compounds Biosynthesis & 2 & 4 \\
\hline -Carbohydrates Biosynthesis & 6 & 9 \\
\hline -Cell Structures Biosynthesis & 4 & 5 \\
\hline -Cofactors, Prosthetic Groups, Electron Carriers Biosynthesis & 34 & 40 \\
\hline -Fatty Acids and Lipids Biosynthesis & 6 & 8 \\
\hline -Metabolic Regulators Biosynthesis & 1 & 1 \\
\hline -Nucleosides and Nucleotides Biosynthesis & 18 & 25 \\
\hline -Other Biosynthesis & 5 & 5 \\
\hline -Secondary Metabolites Biosynthesis & 4 & 3 \\
\hline Degradation/Utilization/Assimilation & 70 & 66 \\
\hline -Alcohols Degradation & 1 & 4 \\
\hline -Amines and Polyamines Degradation & 5 & 3 \\
\hline -Amino Acids Degradation & 18 & 17 \\
\hline -Aromatic Compounds Degradation & 3 & 2 \\
\hline -C1 Compounds Utilization and Assimilation & 1 & 3 \\
\hline -Carbohydrates Degradation & 4 & 2 \\
\hline -Carboxylates Degradation & 5 & 6 \\
\hline -Degradation/Utilization/Assimilation - Other & 2 & 1 \\
\hline -Fatty Acid and Lipids Degradation & 3 & 4 \\
\hline -Inorganic Nutrients Metabolism & 10 & 8 \\
\hline -Nucleosides and Nucleotides Degradation & 6 & 5 \\
\hline -Protein Degradation & 2 & 2 \\
\hline -Secondary Metabolites Degradation & 2 & 2 \\
\hline -Steroids Degradation & 2 & 2 \\
\hline Generation of Precursor Metabolites and Energy & 16 & 25 \\
\hline$\overline{\text { Total }}$ & 185 & 216 \\
\hline
\end{tabular}

\section{Conclusions}

To our knowledge, this is the first comparative genomic study using the complete genome sequences of two C. urealyticum strains, DSM 7109 and DSM 7111. Our analyses provided insights into the genome architecture and the gene content of this species. We found that the C. urealyticum DSM 7111 genome was $50 \mathrm{~kb}$ shorter than the C. urealyticum DSM 7109 genome. This difference in genome size may be linked to the large number of genomic islands (26 for each genome) predicted for both genomes. The genomic islands may have resulted from the horizontal transfer of genes, leading to the acquisition of many strain-specific genes. We detected a high number of strain-specific genes in the two genomes compared with the low number of species-specific genes that have been reported in previous studies of others species of Corynebacterium [25,30]. The horizontal transfer of genes may also explain why $C$. urealyticum is multidrug resistant; i.e., it has received virulence genes by horizontal transfer $[4,5]$.
C. urealyticum is a pathogenic opportunistic bacteria although it showed the spaDEF operon (virulence factor), with a structure similar to that of pathogenic species like $C$. diphtheriae and C. ulcerans. This operon encodes an adhesive pilus responsible for facilitating the adhesion of the pathogen to host cells $[28,43]$.

This comparative genomic study of two C. urealyticum strains provides a basis using reverse vaccinology to predict new antigenic targets. However, additional C. urealyticum strains will have to be studied to create effective vaccines against this bacterium.

\section{Additional material}

Additional file 1: Table S1 C. urealyticum strain-specific pathways.

Competing interests

The authors declare that they have no competing interests. 


\section{Authors' contributions}

LCG, SCS, and VA conceived the study and designed the experiments. LCG, SCS, ET, and RTJR performed the experiments. LCG, SCS, and TRJR analyzed the data. SCS, JB, AS, DB, and VA contributed the materials and analyses tools. AS, DB, JB, and VA provided insights and technical inputs. LCG wrote the paper and SCS, ET, and DB edited the manuscript. All authors read and approved the final manuscript.

\section{Acknowledgements}

We thank the CAPES/PDSE International Cooperation for financing an exchange scholarship (http://www.capes.gov.br/cooperacao-internacional) (grant number 9151125) and Graduate Cluster Industrial Biotechnology (CLIB, http://www.graduatecluster.net/) for support this research.

\section{Declarations}

The publication of this work is funded by CNPq (National Counsel of Technological and Scientific Development), FAPEMIG (Foundation for Research Support of the State of Minas Gerais) and CAPES (Coordination for the Improvement of Higher Education Personnel) number SC-1000675. This article has been published as part of BMC Genomics Volume 16 Supplement 5, 2015: Proceedings of the 10th International Conference of the Brazilian Association for Bioinformatics and Computational Biology (X-Meeting 2014). The full contents of the supplement are available online at http://www.biomedcentral.com/bmcgenomics/supplements/16/S5.

\section{Authors' details}

'Department of General Biology, Institute of Biological Sciences, Federal University of Minas Gerais, Avenue Antônio Carlos, 6627, Belo Horizonte, Minas Gerais, Brazil. ${ }^{2}$ Department of Preventive Veterinary Medicine, School of Veterinary Medicine, Federal University of Minas Gerais, Avenue Antônio Carlos, 6627, Belo Horizonte, Minas Gerais, Brazil. ${ }^{3}$ Institut für Hygiene, Universitätsklinikum Münster, Albert-Schweitzer-Campus 1, Münster, Germany. ${ }^{4}$ Bioinformatics and Systems Biology, Justus-Liebig-University Giessen, Ludwigstasse, 23, Giessen, Germany. ${ }^{5}$ Department of Genetics, Institute of Biological Sciences, Federal University of Pará, Avenue Augusto Corrêa, 01, Belém, Pará, Brazil. ${ }^{6}$ Centre for Genomics and Applied Gene Technology, Institute of Integrative Omics and Applied Biotechnology (IIOAB), Nonakuri, Purba Medinipur, WB-721172, India.

Published: 26 May 2015

\section{References}

1. Soriano F, Ponte C, Santamaria M, Aguado JM, Wilhelmi I, Vela R, Delatte LC: Corynebacterium group D2 as a cause of alkaline-encrusted cystitis: report of four cases and characterization of the organisms. J Clin Microbiol 1985, 21(5):788-792

2. Soriano F, Ponte C, Santamaría M, Castilla C, Fernández Roblas R: In vitro and in vivo study of stone formation by Corynebacterium group D2 (Corynebacterium urealyticum). J Clin Microbiol 1986, 23(4):691-694.

3. Pitcher D, Soto $a$, Soriano F, Valero-Guillén P: Classification of coryneform bacteria associated with human urinary tract infection (group D2) as Corynebacterium urealyticum sp. nov. Int I Syst Bacteriol 1992, 42(1):178-181.

4. Soriano F, Tauch A: Microbiological and clinical features of Corynebacterium urealyticum: urinary tract stones and genomics as the Rosetta Stone. Clin Microbiol Infect 2008, 14(7):632-643.

5. Garcia-Bravo M, Aguado JM, Morales JM, Noriega AR: Influence of external factors in resistance of Corynebacterium urealyticum to antimicrobial agents. Antimicrob Agents Chemother 1996, 40(2):497-499.

6. Tauch A, Trost E, Tilker A, Ludewig U, Schneiker S, Goesmann A, et al: The lifestyle of Corynebacterium urealyticum derived from its complete genome sequence established by pyrosequencing. J Biotechnol 2008, 136(1-2):11-21.

7. Guimarães LC, Soares SC, Albersmeier A, Blom J, Jaenicke S, Azevedo V, et al: Complete Genome Sequence of Corynebacterium urealyticum Strain DSM 7111, Isolated from a 9-Year-Old Patient with Alkaline. 2013, 1(3):2008-2009.

8. Benson D a, Karsch-Mizrachi I, Lipman DJ, Ostell J, Wheeler DL: GenBank. Nucleic Acids Res 2005, 33(Database issue):D34-D38.

9. Gao F, Zhang C-T: Ori-Finder: a web-based system for finding oriCs in unannotated bacterial genomes. BMC Bioinformatics 2008, 9:79.
10. Grissa I, Vergnaud G, Pourcel C: CRISPRFinder: a web tool to identify clustered regularly interspaced short palindromic repeats. Nucleic Acids Res 2007, 35(Web Server issue):W52-W57.

11. Blom J, Albaum SP, Doppmeier D, Pühler A, Vorhölter F-J, Zakrzewski M, Goesmann A: EDGAR: a software framework for the comparative analysis of prokaryotic genomes. BMC Bioinformatics 2009, 10:154.

12. Karp PD, Paley S, Romero P: The Pathway Tools software. Bioinformatics 2002, 18 Suppl 1: S225-S232.

13. Caspi $R$, Altman $T$, Dreher $K$, Fulcher $C a$, Subhraveti $P$, Keseler IM, et al: The MetaCyc database of metabolic pathways and enzymes and the BioCyc collection of pathway/genome databases. Nucleic Acids Res 2012, 40(Database issue):D742-D753.

14. Caspi R, Karp PD: Using the MetaCyc Pathway Database and the BioCyc Database Collection 2007, Chapter 1(December):Unit1.17.

15. Soares SC, Abreu VA, Ramos RT, Cerdeira L, Silva A, Baumbach J, et al: PIPS: pathogenicity island prediction software. PLoS One 2012, 7(2):e30848.

16. Alikhan NF, Petty NK, Ben Zakour NL, Beatson SA: BLAST Ring Image Generator (BRIG): simple prokaryote genome comparisons. BMC Genomics 2011, 12:402.

17. Barh D, Misra AN, Kumar A, Vasco A: A novel strategy of epitope design in Neisseria gonorrhoeae. Bioinformation 2010, 5(2):77-85.

18. Rappuoli R: Reverse vaccinology: a genome-based approach for vaccine development. Vaccine 2001, 17-19: 2688-2691.

19. He Y, Xiang Z, Mobley HL: Vaxign: the first web-based vaccine design program for reverse vaccinology and applications for vaccine development. J Biomed Biotechnol 2010, 2010:297505.

20. Barinov A, Loux V, Hammani A, Nicolas $P$, Langella P, Ehrlich D, et al Prediction of surface exposed proteins in Streptococcus pyogenes, with a potential application to other Gram-positive bacteria. Proteomics 2009, 9(1):61-73.

21. Carver TJ, Rutherford KM, Berriman M, Rajandream MA, Barrell BG, Parkhill J: ACT: the Artemis Comparison Tool. Bioinformatics 2005, 21(16):3422-3423.

22. Hendrickson $\mathrm{H}$, Lawrence JG: Mutational bias suggests that replication termination occurs near the dif site, not at Ter sites. Mol Microbiol 2007, 64(1):42-56.

23. Deveau H, Garneau JE, Moineau S: CRISPR/Cas system and its role in phage-bacteria interactions. Annu Rev Microbiol 2010, 64:475-493.

24. Rasko Da, Rosovitz MJ, Myers GS, Mongodin EF, Fricke WF, Gajer P, et al: The pangenome structure of Escherichia coli: comparative genomic analysis of E. coli commensal and pathogenic isolates. J Bacteriol 2008, 190(20):6881-6893.

25. Ruiz JC, D'Afonseca V, Silva A, Ali A, Pinto AC, Santos AR, et al: Evidence for reductive genome evolution and lateral acquisition of virulence functions in two Corynebacterium pseudotuberculosis strains. PLoS One 2011, 6(4):e18551.

26. Soares SC, Silva A, Trost E, Blom J, Ramos R, Carneiro A, et al: The PanGenome of the Animal Pathogen Corynebacterium pseudotuberculosis Reveals Differences in Genome Plasticity between the Biovar ovis and equi Strains. PLoS One 2013, 8(1):e53818.

27. Schmidt $\mathrm{H}$, Hensel M: Pathogenicity Islands in Bacterial Pathogenesis Pathogenicity Islands in Bacterial Pathogenesis. 2004, 17(1):14-56.

28. Trost E, Blom J, Soares SDC, Huang IH, Al-Dilaimi A, Schröder J, et al: Pangenomic study of Corynebacterium diphtheriae that provides insights into the genomic diversity of pathogenic isolates from cases of classical diphtheria, endocarditis, and pneumonia. J Bacteriol 2012, 194(12):3199-3215

29. Perrin A, Bonacorsi S, Carbonnelle E, Talibi D, Dessen P, Nassif $X$, Tinsley C: Comparative Genomics Identifies the Genetic Islands That Distinguish Neisseria meningitidis, the Agent of Cerebrospinal Meningitis, from Other Neisseria Species Comparative Genomics Identifies the Genetic Islands That Distinguish Neisseria meningitidis. 2002, 70(12):7063-7072

30. Trost E, Al-Dilaimi A, Papavasiliou P, Schneider J, Viehoever P, Burkovski A, et al: Comparative analysis of two complete Corynebacterium ulcerans genomes and detection of candidate virulence factors. BMC Genomics 2011, 12:383.

31. Santos AR, Pereira VB, Barbosa E, Baumbach J, Pauling J, Röttger R, et al: Mature Epitope Density-a strategy for target selection based on immunoinformatics and exported prokaryotic proteins. BMC Genomics 2013, 14(Suppl 6):S4.

32. Mukamolova GV, Turapov O a, Kazarian K, Telkov M, Kaprelyants AS, Kell DB, Young M: The rpf gene of Micrococcus luteus encodes an essential secreted growth factor. Mol Microbiol 2002, 46(3):611-621. 
33. Seyffert N: Isolamento, identificação e análise molecular do gene cpsec39 codificador de uma proteína exclusiva de Corynebacterium pseudotuberculosis: perspectivas no desenvolvimento de métodos profiláticos para o controle da linfadenite caseosa. Federal University of Minas Gerais 2009

34. Chambaud I, Wróblewski H, Blanchard A: Interactions between mycoplasma lipoproteins and the host immune system. Trends Microbiol 1999, 7(12):493-499.

35. Broadway MM, Rogers EA, Chang C, Huang $\mathrm{I}-\mathrm{H}$, Dwivedi $\mathrm{P}$, Yildirim S, et al: Pilus gene pool variation and the virulence of Corynebacterium diphtheriae clinical isolates during infection of a nematode. J Bacteriol 2013, 195(16):3774-3783

36. Baker JL, Sudarsan N, Weinberg Z, Roth A, Stockbridge RB, Breaker RR: Widespread genetic switches and toxicity resistance proteins for fluoride. Science 2012, 335(6065):233-235.

37. Mukamolova GV, Turapov OA, Young DI, Kaprelyants AS, Kell DB, Young M: A family of autocrine growth factors in Mycobacterium tuberculosis. Mol Microbiol 2002, 46(3):623-635

38. Marcyjaniak M, Odintsov SG, Sabala I, Bochtler M: Peptidoglycan amidase MepA is a LAS metallopeptidase. J Biol Chem 2004, 279(42):43982-43989.

39. Tomioka S, Matsuhashi M: Purification of penicillin-insensitive DDendopeptidase, a new cell wall peptidoglycan-hydrolyzing enzyme in Escherichia coli, and its inhibition by deoxyribonucleic. Biochem Biophys Res 1978, 84(4):978-984.

40. Guimarães LC, Silva NF, Miyoshi A, Schneider MPC, Silva A, Azevedo V, et al: Structure modeling of a metalloendopeptidase from Corynebacterium pseudotuberculosis. Comput Biol Med 2012, 42(5):538-541.

41. Sutcliffe IC, Harrington DJ: Lipoproteins of Mycobacterium tuberculosis: an abundant and functionally diverse class of cell envelope components. FEMS Microbiol Rev 2004, 28(5):645-659.

42. Schröder J, Maus I, Meyer K, Wördemann S, Blom J, Jaenicke S, et al: Complete genome sequence, lifestyle, and multi-drug resistance of the human pathogen Corynebacterium resistens DSM 45100 isolated from blood samples of a leukemia patient. BMC Genomics 2012, 13:141.

43. Gaspar AH, Ton-That H: Assembly of Distinct Pilus Structures on the Surface of Corynebacterium diphtheriae Assembly of Distinct Pilus Structures on the Surface of Corynebacterium diphtheriae. 2006, 188(4):1526-1533.

44. Karp PD, Paley SM, Krummenacker M, Latendresse M, Dale JM, Lee TJ, et al: Pathway Tools version 13.0: integrated software for pathway/genome informatics and systems biology. Briefings Bioinforma 2010, 11(1):40-79.

doi:10.1186/1471-2164-16-S5-S7

Cite this article as: Guimarães et al:: Genome informatics and vaccine targets in Corynebacterium urealyticum using two whole genomes, comparative genomics, and reverse vaccinology. BMC Genomics 2015 16(Suppl 5):S7.

\section{Submit your next manuscript to BioMed Central and take full advantage of:}

- Convenient online submission

- Thorough peer review

- No space constraints or color figure charges

- Immediate publication on acceptance

- Inclusion in PubMed, CAS, Scopus and Google Scholar

- Research which is freely available for redistribution

Submit your manuscript at www.biomedcentral.com/submit
Biomed Central 